\title{
CAPÍTULO 01: ANÁLISE DA VARIAÇÃO DO PERFIL EPIDEMIOLÓGICO DA SÍFILIS GESTACIONAL E SUA PREVALÊNCIA EM UM MUNICÍPIO DO ESTADO DE PERNAMBUCO
}

\section{CAPÍTULO 01: ANÁLISIS DE LA VARIACIÓN DEL PERFIL EPIDEMIOLÓGICO DE LA SÍFILIS GESTACIONAL Y SU PREVALENCIA EN UN MUNICIPIO DEL ESTADO DE PERNAMBUCO}

\section{CHAPTER 01: ANALYSIS OF THE VARIATION OF THE EPIDEMIOLOGICAL PROFILE OF GESTATIONAL SYPHILIS AND ITS PREVALENCE IN A MUNICIPALITY IN THE STATE OF PERNAMBUCO}

Damiana Maria Minhaqui da Conceição후 Joyce Santana do Nascimento²; Angela Maria Leal de Moraes Vieira ${ }^{3}$.

DOI: $\underline{\text { https://doi.org/10.31692/978-65-88970-04-1.1-14 }}$

\begin{abstract}
RESUMO
A sífilis, causada pela bactéria Treponema pallidum, transmitida através da relação sexual e também de forma vertical (mãe-filho), sofre influência dos aspectos socioeconômicos para a não adesão do tratamento em virtude da ausência ou do início tardio do pré-natal, como é o caso do município de Recife, no estado de Pernambuco. Nesta visão, o objetivo do presente trabalho é identificar a relação do perfil epidemiológico da sífilis gestacional (SG) no município de Recife e a influência das principais variáveis encontradas. Tratando-se de um estudo de carácter descritivo, a análise dos dados ocorreu a partir de dados secundários com base no Sistema de Informação de Agravos de Notificação - SINAN. O universo foi composto pela região do município de Recife-Pe, dispondo critérios de inclusão apenas mulheres acometidas, sendo excluídos as demais classificações da doença, outras localidades não correspondentes a região em estudo e anos anteriores a 2009 no Sinan. Após a coleta e análise dos dados entre os anos de 2009-2019, a variável relacionada a faixa etária da SG no município do Recife demonstrou uma maior prevalência de casos entre 20 a 29 anos de idade. A idade gestacional apresentou uma grande necessidade para a detecção oportuna das mulheres acometidas, sendo observado no $1^{\circ}$ trimestre uma taxa de detecção de apenas 52 casos em 2019, diferentemente do que acontece no $3^{\circ}$ trimestre do mesmo ano, o qual apresentou o registro de 177 casos. Quanto ao padrão raça/cor da pele, houve a maior prevalência entre mulheres autodeclaradas pardas, apresentando o total de 1.396 casos. Com relação ao nível de escolaridade, foi possível notar que quanto maior o nível escolaridade, menor o número de mulheres gestantes diagnosticadas com sífilis. Já a notificação de acordo com a classificação clínica, o estágio primário da doença foi apontado como o de maior registro de notificação, correspondendo a um total de 639 casos. Dessa forma, pode-se perceber que o registro de casos notificados no ano de 2019 apresentou uma queda em comparação ao ano de 2018. Assim, são necessárias a captação precoce e a adesão da gestante ao pré-natal, assim como uma assistência de qualidade, fazendo com que haja a continuidade da redução dos casos de gestantes com sífilis no município de Recife-Pe.

Palavras-Chave: Gestantes, Prevenção, Notificação, Sífilis.

\section{RESUMEN}

La sífilis, causada por la bacteria Treponema pallidum, transmitida a través de las relaciones sex también de manera vertical (madre-hijo), está influenciada por aspectos socioeconómicos por la adherencia al tratamiento por ausencia o inicio tardío del prenatal, como este es el caso del $\mathrm{m}$ de Recife, en el estado de Pernambuco. En esta perspectiva, el objetivo del presente estudio es la relación entre el perfil epidemiológico de la sífilis gestacional (SG) en la ciudad de influencia de las principales variables encontradas. En el caso de un estudio descriptiy
\end{abstract}

${ }^{1}$ Enfermagem, Centro Universitário São Miguel (UNISÃOMIGUEL), damianamin

2 Enfermagem, Centro Universitário São Miguel (UNISÃOMIGUEL), joyce.jsn8@y

${ }^{3}$ Mestre, Centro Universitário São Miguel (UNISÃOMIGUEL), am.enfermagem@gmail.com 
los datos se dio a partir de datos secundarios con base en el Sistema de Informação de Agravos de Notificação - SINAN (Sistema de Información de Enfermedades Notificables). El universo estuvo constituido por la región de la ciudad de Recife-PE, con criterios de inclusión solo para mujeres afectadas, excluyendo las otras clasificaciones de la enfermedad, otras localizaciones no correspondientes a la región en estudio y años anteriores al 2009 en Sinan. Después de recolectar y analizar datos entre los años 2009-2019, la variable relacionada con el grupo de edad de SG en la ciudad de Recife mostró una mayor prevalencia de casos entre los 20 y 29 años. La edad gestacional mostró una gran necesidad de detección oportuna de las mujeres afectadas, con una tasa de detección de solo 52 casos en 2019 en el primero trimestre, a diferencia de lo que ocurre en el tercero trimestre del mismo año, que registró 177 casos. En cuanto al patrón de raza / color de piel, hubo una mayor prevalencia entre las mujeres autodeclaradas de piel morena, con un total de 1396 casos. En cuanto al nivel de educación, se pudo notar que a mayor nivel de educación, menor es el número de embarazadas diagnosticadas con sífilis. En cuanto a la notificación según la clasificación clínica, la etapa primaria de la enfermedad se identificó como la de mayor registro de notificación, correspondiente a un total de 639 casos. Así, se puede observar que el registro de casos notificados en el año de 2019 presentó una disminución en comparación con el año de 2018. Por ello, es necesaria la captación temprana y adherencia de la gestante a el prenatal, así como una atención de calidad, provocando la continuidad de la reducción de casos de mujeres embarazadas con sífilis en la ciudad de Recife-PE.

Palabras Clave: Embarazada, prevención, notificación, sífilis.

\section{ABSTRACT}

Syphilis, caused by the bacteria Treponema pallidum, transmitted through sexual intercourse and also vertically (mother-child), is affected by socioeconomic aspects for non adherence to treatment due to the absence or late onset of prenatal, as it is the case of Recife, Pernambuco state. In this manner, the intention of the present work is to identify the epidemiological profile of gestational syphilis (GS) in the city of Recife and its influences on the main variables found. As a descriptive study, the analysis of the data presented originated from secondary data based on the SINAN - Sistema de Informação de Agravos de Notificação. The universe was composed by the Recife-Pe region, having as an inclusion criterion only women who had been affected, excluding the other classifications of the disease, locations not corresponding to the region under analysis and time before 2009 at SINAN. After data collection and analysis in the years between 2009-2019, the variable related to the GS age group in the city of Recife showed a higher prevalence of cases in the ages between 20-29 years old. The gestational age showed a great need for instant detection of affected women, with a detection rate of only 52 cases in 2019 in the 1st quarter, unlike in the 3rd quarter of the same year, which showed 177 cases. As for the race/skin color criteria, there was the highest prevalence among self-declared brown women, presenting a total of 1,396 cases. Regarding the level of schooling, it was possible to notice that the higher the schooling level, the lower the number of pregnant women diagnosed with syphilis. On the other hand, according to the clinical classification, the primary stage of the disease was indicated as the one with the highest notification record, corresponding to a total of 639 cases. Thus, it can be seen that the register of notified cases in the year 2019 presented a decrease compared to the year of 2018. Thus, early detection and adherence of pregnant women to prenatal care are necessary, as well as quality care, leading to a continued reduction in cases of pregnant women with syphilis in the city of Recife-Pe.

Keywords: Pregnant, Prevention, Notification, Syphilis.

\section{INTRODUÇÃO}

A saúde sexual é um direito garantido pelo Estado através das Internacionais que aconteceram em outrora e deram base para a elaboração de políticas voltadas para a saúde sexual e reprodutiva. É assegurado, dentre outros direitos, o direito ao planejamento reprodutivo de forma segura, de maneira que o usuário da rede de saúde tenha 
autonomia para decidir quando e quais os melhores métodos possa utilizar. Assim, a prevenção e o controle das Infecções Sexualmente Transmissíveis (IST) devem acontecer por intermédio dos profissionais de saúde. Todavia, nota-se ainda casos passíveis de prevenção sendo recorrentes no século XXI (BRASIL, 2013).

Considerada uma IST, a sífilis é uma doença bacteriana causada pelo Treponema pallidum, podendo ser transmitida através da relação sexual desprotegida, transfusão sanguínea e de forma vertical (mãe-filho), por exemplo. A patologia é conhecida desde o século XV na Europa, sendo seu marco no tratamento o desenvolvimento da penicilina. Assim, com o surgimento do fármaco, os casos de sífilis passaram a ser controlados, levando a falsa impressão da erradicação da doença. (AVELLEIRA; BOTTINO, 2006).

Com o surgimento da pílula anticoncepcional, os casos de sífilis voltaram a ter expressividade por volta de 1960, momento em que a transmissão do HIV passa a ser um fator facilitador em virtude da infecção pela sífilis e a doença passa a receber uma maior atenção novamente. A sífilis é a IST mais comum encontrada nos pacientes portadores de HIV/AIDS, sendo a coinfecção HIV/Sífilis mais encontrada em gestantes (ACOSTA; GONÇALVES; BARCELLOS, 2016).

No Brasil, o Sistema Único de Saúde (SUS) preconiza a prevenção da ocorrência desse evento sentinela oferecendo diagnóstico e tratamento gratuitos para a população, com destaque para as políticas públicas direcionadas às gestantes e seus parceiros sexuais. No entanto, um crescente número de casos de sífilis congênita, óbitos fetais, abortos e diversas sequelas irreversíveis para os recém-nascidos surgiram em decorrência dessa infecção pela falta de tratamento (LAZARINI; BARBOSA, 2017).

A região Nordeste ocupa o segundo lugar do ranking das regiões com maior número de casos de sífilis em gestantes no país, sendo o estado do Maranhão o que apresenta a maior taxa de incidência dessa região, com seis casos por mil nascidos vivos, concentrando as maiores taxas em São Luís, capital do estado, seguido pelos municípios de Imperatriz, Codó Caxias (CONCEIÇÃO, 2019).

A Organização Mundial da Saúde (OMS) estima que ocorra aproximadamente milhão de infecções por sífilis gestacional (SG), sendo mais atingidas mulheres com níveis socioeconômicos reduzidos e estilos de vida que contribuem para a infecção,

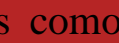
múltiplos parceiros, resistência ao uso de preservativos, além de apresentar antecedentes obstétricos de risco, início tardio do acompanhamento pré-natal, redução do número de consultas, tratamento inadequado dos casos diagnosticados e o não (RAMOS, 2018) 
Tendo em vista a sífilis como uma patologia favorável a prevenção, medidas educativas em saúde devem ser colocadas em prática, associado ao mapeamento das regiões mais afetadas, fazendo com que haja o maior acompanhamento e conhecimento dos profissionais de saúde, especialmente da atenção primária, no diagnóstico precoce e tratamento efetivo das gestantes situadas no Recife. Dessa forma, prevenindo possíveis sequelas para o recém-nascido $(\mathrm{RN})$ e para a mulher.

Frente ao cenário epidemiológico da SG, a falta de informação e educação sexual corroboram para os dados e tabelas gerados a partir das notificações realizadas no Sistema de Informação de Agravos de Notificação - SINAN. Desse modo, possíveis complicações relativas a esse agravo justificam a relevância em descrever a prevalência dos casos de SG no Recife, cooperando no desfecho favorável de uma assistência de qualidade e adesão de ações e estratégias efetivas no controle e erradicação dessa condição patológica evitável e tratável.

Sendo assim, diante do panorama atual e da relevância na abordagem do tema, emergem questionamentos acerca do perfil epidemiológico das mulheres com sífilis gestacional localizadas no município do Recife, estado de Pernambuco, tais como: faixa etária, idade gestacional, nível de escolaridade, raça/cor e classificação clínica da doença.

Diante do exposto, objetivo do presente trabalho é identificar a relação do perfil epidemiológico da sífilis gestacional (SG) no município de Recife e a influência das principais variáveis encontradas.

\section{FUNDAMENTAÇÃO TEÓRICA}

A sífilis na gestação é de notificação compulsória desde 2005, sendo os casos suspeitos definidos quando a gestante apresenta evidências clínicas ou teste não treponêmico reagente, e caso confirmado quando o teste não treponêmico e o teste treponêmico for reagente em qualquer titulação, tendo ou não manifestações clínicas. Com o maior alcance da vigilância epidemiológica, a testagem rápida no pré-natal obteve maior cobertura, apontando 21.382 novos casos de sífilis em gestantes no Sinan no ano de 2013 (BRASIL, 2015).

A sífilis pode ser classificada como primária, secundária e terciária, possuindo fases diversificadas e períodos de latência. A sífilis primária tem como lesão específica o cancro duro, o qual se desenvolve no local da inoculação em um período de três semanas posteriores a ocorrência da infecção. Após um período de latência, característico com yariações de seis a oito semanas, a bactéria dissemina-se pelo corpo (GUANABARA et al., 2014).

A sífilis secundária tem sua ocorrência em um período posterior que vai de seis 
semanas a seis meses da infecção primária que não foi tratada, com o aparecimento de lesões papulosas que podem acometer as regiões palmar e plantar. $\mathrm{Na}$ fase terciária ocorre o aparecimento de lesões que se localizam nas mucosas, na pele, nos sistemas nervoso e cardiovascular. $\mathrm{O}$ aspecto principal dessa fase se dá com a formação de granulomas destrutivos (BERNARDES FILHO et al., 2012).

A sífilis primária foi prevalente nas análises epidemiológicas da sífilis gestacional no Brasil, entretanto, a observância do predomínio dessa fase clínica, em grande parte do preenchimento das fichas, pode estar relacionada à ausência de conhecimento dos profissionais sobre a infecção, ou seja, o preenchimento pode ter sido feito de maneira equivocada. De acordo com a fisiopatologia da infecção e da prevalência da fase latente, a sífilis dificilmente é diagnóstica na fase primária (CONCEIÇÃO, 2019).

Quando acomete a gestante, a sífilis pode provocar a sífilis congênita (SC), que é responsável por aproximadamente $40 \%$ das taxas de mortalidade perinatal, $25 \%$ de mortalidade e $14 \%$ de mortes neonatais, acarretando graves consequências para o concepto (CARDOSO; ARAÚJO, et al., 2018).

Nas gestantes portadoras da infecção, a doença pode apresentar complicações como o aborto espontâneo, prematuridade, má-formação do feto, surdez, morte do neonato ou a morte intraútero, além de baixo peso, rinite com coriza sanguinolenta, obstrução nasal, prematuridade, choro ao manuseio, hepatoesplenomegalia, alterações respiratórias como pneumonia, icterícia, anemia severa, ascite e lesões cutâneas na palma da mão e no pé (RAMPAZIO et al., 2019).

No Brasil, entre 2010 a 2018 verificou-se uma evolução na taxa de incidência de sífilis gestacional, havendo um aumento de 3,8 vezes, passando de 2,4 para 9,0 casos por mil nascidos vivos, e a taxa de detecção de sífilis em gestantes aumentou 6,1 vezes, passando de 3,5 para 21,4 casos por mil nascidos vivos. Segundo a Organização Mundial da Saúde (OMS), os números de casos da infecção são preocupantes e precisam ser controlados (BRASIL 2019).

Alguns fatores estão diretamente ligados a ocorrência da SG, tais como: baixo nível de escolaridade, raça/cor, precária condição socioeconômica, diversidade de parceiros captação tardia da gestante e o número insuficiente de consultas pré-natais (FURTADO et al. 2017). Paralelo a isso, a sífilis vem apresentando um crescimento exponencial em sua incidência, que pode ser resultado da escassez de cuidados durante o ato sexual, bem como da maior efetividade do processo gerencial das notificações nos sistemas de vigilância epidemiológica (ARAÚJO et al., 2016). 
No estado de Pernambuco, um estudo publicado em 2019, permitiu identificar 1.494 casos de adolescentes com SG entre os anos de 2007 a 2016, o que demonstrou um aumento de $407 \%$ quando comparado os anos de 2007 e 2016 . A idade gestacional mais prevalente foi o $2^{\circ}$ trimestre com adolescentes entre 15 e 19 anos de idade, sendo a raça/cor parda mais expressiva no período análise, assim como o nível de escolaridade da $5^{\mathrm{a}}$ à $8^{\mathrm{a}}$ série incompleta do ensino fundamental mais evidente no estudo (OLIVEIRA; PEIXOTO; CARDOSO, 2019).

Ainda segundo o estudo anterior, a região de saúde mais afetada no período pela SG na adolescência foi a regional I, com ênfase nos municípios de Recife, Olinda e Jaboatão dos Guararapes, regional IV e VIII, as quais corresponderam a 13,7\% e 5,7\% dos casos, respectivamente. Apenas no município de Recife, 779 (52,1\%) casos foram registrados entre 2007-2016, podendo o nível de escolaridade ter influenciado no índice elevado das adolescentes acometidas, bem como o não tratamento (OLIVEIRA; PEIXOTO; CARDOSO, 2019).

Um estudo do tipo caso-controle realizado com gestantes de 7 (sete) maternidades do Recife, apontou a relação entre a sífilis e a pobreza, consequentemente com os fatores de vulnerabilidades decorrentes da situação socioeconômica. Dentre as problemáticas relatadas, foi indicada a não realização do pré-natal e sua baixa realização pelas gestantes como um fator causador de complicações ao longo da gravidez, assim como o início precoce de relações sexuais como um risco maior a saúde (MACÊDO et al., 2016).

Além disso, a sífilis em gestantes se correlaciona com a sua situação sociodemográfica desvantajosa e fatores socioeconômicos. Ambos, muitas vezes estão associados a um escasso, longínquo e superlotado acesso de saúde, com atendimento inadequado e ineficaz, e estes têm sido fatores contribuintes para disseminação da infecção (OLIVEIRA et al., 2020).

Apesar de avanços no SUS, o combate à SC com base no tratamento da sífilis gestacional permanece como desafio, sobretudo ao observar o seu aumento ao longo dos anos e por considerarmos que a ocorrência da SC indica fragilidades na atenção ao pré-natal, sendo portanto, um evento sentinela para o monitoramento do acesso e da qualidade da atenção básica (FIGUEIREDO et al., 2020).

\section{METODOLOGIA}

Trata-se de um estudo de carácter descritivo realizado a partir secundários, pertencentes ao banco de dados do Sistema de Informação de Agravos de Notificação - SINAN, que tem como objetivo coletar, transmitir e disseminar dados gerados 
rotineiramente pelo Sistema de Vigilância Epidemiológica das três esferas de governo, por intermédio de uma rede informatizada. Acrescidos de dados do Ministério da Saúde (MS), e Artigos Científicos.

Por ser um estudo que analisa dados secundários e por tratar-se de informações de domínio público, conforme a Resolução 466/12, dispensam a análise e aprovação do Comitê de Ética em Pesquisa.

Essa metodologia se aplica quando alguns estudos questionam as justificativas devida à possibilidade da realização de pesquisas sem o consentimento informado dos sujeitos da pesquisa ou nos casos em que os indivíduos não mantêm contato com os realizadores e não participam fisicamente do estudo.

Esse método de análise permite investigar o contexto de saúde em duas vertentes: a condição epidemiológica da doença, no tocante aos grupos de acometidos, e a distribuição geográfica no período de ocorrência.

O universo caracterizado para efeito desta investigação foi composto por dados selecionados da região do Recife, PE. Como critérios de inclusão, apenas mulheres com sífilis gestacional localizadas no Recife foram selecionadas, utilizando-se de critérios de exclusão as demais classificações de sífilis, outras localidades não correspondentes a região em estudo e anos anteriores a 2009 no Sinan.

Os resultados foram coletados a partir das planilhas disponibilizadas no SINAN, sistema produzido para análises estatísticas e epidemiológicas, e seus dados foram analisados no programa Excel versão Office 2019.

\section{RESULTADOS E DISCUSSÃO}

Foram analisadas as principais incidências relacionadas a sífilis na gestação, a começar pela Faixa Etária (descrita na Tabela 1), Idade Gestacional (descrita na Tabela 2), Raça/Cor (descrita na Tabela 3), Escolaridade (descrita na Tabela 4) e Classificação clínica (descrita na Tabela 5). Devido ao grande contingente de dados coletados no Sistema (SINAN), foi realizado a tabulação desses dados.

A análise da investigação sobre os fatores associados à sífilis gestacional em/mulheres demonstrou que a doença se encontra fortemente associada as condições de vulnerabilidade, sejam comportamentais ou de acesso a qualidade da assistência ofertada nos

Nas tabelas a seguir, serão analisadas as populações de mulheres infectadas por Sífilis na Gestação no município do Recife. 
Tabela 1. Casos de gestantes com sífilis segundo faixa etária por ano de diagnóstico. Dados estatísticos para Sífilis - Recife. 2009-2019

\begin{tabular}{cccccccccccccc}
\hline $\begin{array}{c}\text { Faixa } \\
\text { Etária }\end{array}$ & Total & $\mathbf{2 0 0 9}$ & $\mathbf{2 0 1 0}$ & $\mathbf{2 0 1 1}$ & $\mathbf{2 0 1 2}$ & $\mathbf{2 0 1 3}$ & $\mathbf{2 0 1 4}$ & $\mathbf{2 0 1 5}$ & $\mathbf{2 0 1 6}$ & $\mathbf{2 0 1 7}$ & $\mathbf{2 0 1 8}$ & $\mathbf{2 0 1 9}$ \\
\hline $\begin{array}{c}\text { 10 a 14 } \\
\text { anos }\end{array}$ & 37 & 2 & - & 1 & 2 & 4 & 5 & 2 & 2 & 7 & 9 & 3 \\
\hline $\begin{array}{c}\text { 15 a 19 } \\
\text { anos }\end{array}$ & 581 & 22 & 18 & 20 & 32 & 36 & 47 & 50 & 53 & 70 & 176 & 57 \\
\hline $\begin{array}{c}\text { 20 a 29 } \\
\text { anos }\end{array}$ & 1.373 & 54 & 49 & 64 & 70 & 80 & 93 & 98 & 88 & 178 & 423 & 176 \\
\hline $\begin{array}{c}\text { 30 a 39 } \\
\text { anos }\end{array}$ & 499 & 16 & 31 & 24 & 33 & 25 & 33 & 31 & 26 & 51 & 149 & 80 \\
\hline $\begin{array}{c}\text { 40 anos } \\
\text { ou mais }\end{array}$ & 40 & 1 & 1 & 2 & 2 & 1 & 3 & 3 & 4 & 5 & 13 & 5 \\
\hline Ignorado & - & - & - & - & - & - & - & - & - & - & - & - \\
\hline
\end{tabular}

A faixa etária mostrou-se associada aos casos de gestantes com sífilis, embora se observe uma tendência de mudança do perfil etário, com disseminação do agravo em todas as faixas. As pesquisas nos últimos dez anos demonstraram a prevalência de mulheres com idade média de 20 a 29 anos. Com isso, tem-se a necessidade de ampliar o desenvolvimento de ações de educação e prevenção para alcançar as mulheres de todas as faixas etárias, incluindo as mulheres de maior vulnerabilidade e da faixa etária dos 10 aos 19 anos de idade.

Apesar da faixa etária entre 15 e 19 anos de idade representar o segundo maior quantitativo de casos entre os anos de 2009-2019, poucos são os estudos voltados para a adolescente gestante com sífilis. Do total de casos registrados neste período, 581, o ano de 2018 obteve maior taxa, correspondendo a 176 (30,29\%) casos, diferentemente do ano de 2019 que apresentou uma redução de 67,61\% dos casos em comparação a 2018.

Entre 30 e 39 anos, a faixa etária de mulheres diagnosticados com SG no município de Recife teve um total de 499 casos notificados, sendo 2018 apontado como o ano de maior registro, $149(29,85 \%)$ casos, e 2019 registrando 80 (16,03\%) casos, uma redução de 69 ocorrências. Em 2018, as mulheres com 40 anos ou mais também apresentaram a SG, sendo registrado 40 casos no total. As mulheres nessa faixa etária devem ser acompanhadas e testadas a fim de se evitar uma gestação de alto risco relacionada a idade e a SG.

A falta de conhecimento e informação sobre os riscos da doença, formas de prevenção e contágio vem colaborando para a reinfecção, com isso elevando o número gestantes, torna-se ainda mais complicada a infecção ou reinfecção da sífilis. Todas as gestantes devem ser substancialmente informadas, orientadas e questionadas sobre os riscos de uma vida sexual sem segurança advinda da falta de métodos preventivos e suas consequências. 
Tabela 2. Casos de gestantes com sífilis segundo idade gestacional por ano de diagnóstico. Dados estatísticos para Sífilis - Recife. 2009-2019

\begin{tabular}{|cccccccccccccc}
\hline $\begin{array}{c}\text { Idade } \\
\text { Gestacional }\end{array}$ & Total & $\mathbf{2 0 0 9}$ & $\mathbf{2 0 1 0}$ & $\mathbf{2 0 1 1}$ & $\mathbf{2 0 1 2}$ & $\mathbf{2 0 1 3}$ & $\mathbf{2 0 1 4}$ & $\mathbf{2 0 1 5}$ & $\mathbf{2 0 1 6}$ & $\mathbf{2 0 1 7}$ & $\mathbf{2 0 1 8}$ & $\mathbf{2 0 1 9}$ \\
$\begin{array}{c}\mathbf{1}^{\circ} \\
\text { Trimestre }\end{array}$ & 414 & 14 & 16 & 16 & 13 & 19 & 37 & 40 & 37 & 55 & 115 & 52 \\
\hline $\begin{array}{c}\mathbf{2}^{\circ} \\
\text { Trimestre }\end{array}$ & 880 & 49 & 41 & 52 & 62 & 57 & 78 & 92 & 79 & 118 & 176 & 76 \\
\hline $\begin{array}{c}\mathbf{3}^{\circ} \\
\text { Trimestre }\end{array}$ & 1.103 & 29 & 39 & 36 & 55 & 61 & 51 & 43 & 50 & 115 & 447 & 177 \\
\hline $\begin{array}{c}\text { Idade } \\
\text { gestacional } \\
\text { ignorada }\end{array}$ & 133 & 3 & 3 & 7 & 9 & 9 & 15 & 9 & 7 & 23 & 32 & 16 \\
\hline Ignorado & 0 & - & - & - & - & - & - & - & - & - & - & - \\
\hline & Fonte: Sistema de Informação de Agravos de Notificação-SINAN & & \\
\hline
\end{tabular}

Observa-se que o $3^{\circ}$ trimestre da gestação é o período em que há o maior quantitativo de casos de gestantes com sífilis, sendo a partir do ano de 2017 que passa a ocorrer um sobressalto em relação ao $1^{\circ}$ e $2^{\circ}$ trimestre. Tal evidência demonstra a necessidade do acompanhamento destas mulheres no pré-natal para a realização de testes rápidos que possam promover o diagnóstico e o tratamento precoce, fazendo com que possíveis sequelas decorrentes da doença venham a ser prevenidas no binômio mãe-filho.

O diagnóstico da infecção no terceiro trimestre gestacional é considerado tardio e pode estar relacionado ao início tardio do pré-natal nas gestantes, bem como a baixa sensibilidade e efetividade da assistência pré-natal oferecida. Esses resultados reafirmam a importância da detecção oportuna das gestantes com sífilis, assim como da oferta de oportunidades de tratamento correto para as gestantes e seus parceiros.

É observada na tabela uma queda significativa no número de casos registrados no $3^{\circ}$ trimestre da gravidez no ano de 2018, o qual passou de 447 (40,52\%) para $177(16,04 \%)$ em 2019, redução tal que representou uma baixa de $270(60,40 \%)$ novos casos. O $1^{\circ}$ trimestre também obteve uma diminuição no número de gestantes com sífilis em 2018, passando de $(27,7 \%)$ casos em 2018 para $52(12,56 \%)$ em 2019. O mesmo acontece com as gestantes diagnosticadas no $2^{\circ}$ trimestre, obtendo-se uma diferença de $100(56,81 \%)$ novos casos quando comparados os anos de 2018 e 2019.

Quanto ao padrão raça/cor da pele, este estudo vai de encontro aos citados pesquisa na capital de Pernambuco, Recife, entre os anos de 2009 a 2019, cuja maior predominância foi entre mães autodeclaradas pardas, com um total 1.396 casos entre os anos

Os dados da tabela demonstram a população parda como a mais afetada entre os anos 
de 2009 a 2019. Em 2011, o menor número de casos foi registrado, 53 (3,79\%), diferente do que aconteceu no ano de 2018, sendo registrada a maior taxa, 418 (29,94\%). Todavia, o ano de 2019 teve uma queda na taxa de infectadas, correspondendo a $195(13,96 \%)$ gestantes com sífilis.

Tabela 3. Casos de gestantes com sífilis segundo raça ou cor por ano de diagnóstico. Recife, 2009-2019

\begin{tabular}{lcccccccccccc}
\hline $\begin{array}{c}\text { Raça ou } \\
\text { Cor }\end{array}$ & Total & $\mathbf{2 0 0 9}$ & $\mathbf{2 0 1 0}$ & 2011 & 2012 & 2013 & 2014 & 2015 & 2016 & 2017 & 2018 & 2019 \\
\hline Branca & 296 & 10 & 13 & 10 & 19 & 19 & 19 & 23 & 28 & 32 & 83 & 40 \\
\hline Preta & 266 & 10 & 11 & 16 & 8 & 9 & 16 & 22 & 15 & 43 & 86 & 30 \\
\hline Amarela & 16 & 2 & 1 & 1 & - & 2 & 2 & 1 & - & 1 & 4 & 2 \\
\hline Parda & 1.396 & 61 & 61 & 53 & 83 & 71 & 89 & 98 & 89 & 178 & 418 & 195 \\
\hline Indígena & 2 & - & - & - & - & - & - & - & - & 1 & 1 & - \\
\hline Ignorada & 554 & 12 & 13 & 31 & 29 & 45 & 55 & 40 & 41 & 56 & 178 & 54 \\
\hline
\end{tabular}

Fonte: Sistema de Informação de Agravos de Notificação - SINAN

A população autodeclarada branca e preta tiveram dados aproximados. Enquanto as mulheres brancas com SG tiveram um total de 296 casos registrados, as mulheres pretas tiveram o total de 266 registros. No ano de 2009 , população branca e preta apresentaram a mesma taxa de notificação, 10 casos, correspondendo a um percentual de 3,37\% e 3,75\%, respectivamente.

O ano de 2018 apresentou as taxas mais elevadas, 83 (28,04\%) mulheres brancas com SG no Recife e 86 (32,33\%) mulheres pretas com SG no mesmo município. Entretanto, em 2019, houve a diminuição da SG entre mulheres consideradas brancas para $40(13,51 \%)$ casos e entre a mulheres autodeclaradas pretas, $30(11,27 \%)$ casos registrados.

As mulheres autodeclaradas amarelas e indígenas obtiveram as menores taxas registradas nos últimos 10 anos (2009-2019). Apenas 2 casos foram registrados na população indígena, 1 caso em 2017 e outro em 2018, contudo, a ausência de registros não significa a ausência de casos. Em relação as mulheres autodeclaradas amarelas, foi registrado um total de 16 casos de mulheres com SG entre 2009-2019, sendo os anos de 2012 e 2016 os únicos com ausência de casos.

O estudo revelou que, no estado de Pernambuco, o grau de escolaridade das mulheres mais atingidas pela doença esteve entre a $5^{\mathrm{a}}$ e $8^{\mathrm{a}}$ série, sendo responsável por uma elevada porcentagem. A escolaridade parece ter o seu destaque reduzido nas práticas de risco as IST uma vez que, independente de escolaridade, atualmente a população não tem acesso considerável à informação básica sobre as formas de transmissão dessas doene

É apontado na tabela a relação entre o baixo nível de escolaridade com a maior vulnerabilidade das gestantes localizadas no Recife em adquirir a sífilis. Pode-se notar a maior 
prevalência de casos entre a $5^{\text {a }}$ e $8^{\text {a }}$ série incompleta, 540 casos entre os anos de 2009 a 2019. Novamente, é destacado o ano de 2018 como o ano mais discrepante nos registros de novos casos, 172 (31,85\%), e o ano de 2019 como o ano de redução de casos, 74 (13,70\%).

Tabela 4. Casos de gestantes com sífilis segundo escolaridade por ano de diagnóstico. Recife, 2009-2019

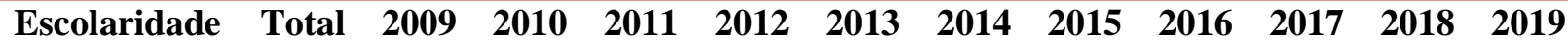

\begin{tabular}{|c|c|c|c|c|c|c|c|c|c|c|c|c|}
\hline Analfabeto & 22 & 3 & 5 & 2 & 1 & - & 2 & 2 & 1 & 2 & 2 & 2 \\
\hline $\begin{array}{l}1^{\mathrm{a}} \text { a } 4^{\mathrm{a}} \text { série } \\
\text { incompleta }\end{array}$ & 116 & 7 & 9 & 11 & 6 & 5 & 10 & 10 & 9 & 11 & 28 & 10 \\
\hline $\begin{array}{l}4^{\mathrm{a}} \text { série } \\
\text { completa }\end{array}$ & 100 & 8 & 8 & 6 & 10 & 4 & 9 & 7 & 4 & 9 & 24 & 11 \\
\hline $\begin{array}{l}5^{\mathrm{a}} \text { a } 8^{\mathrm{a}} \text { série } \\
\text { incompleta }\end{array}$ & 540 & 15 & 18 & 21 & 35 & 29 & 31 & 46 & 31 & 68 & 172 & 74 \\
\hline $\begin{array}{l}\text { Fundamental } \\
\text { Completo }\end{array}$ & 143 & 6 & 6 & 5 & 10 & 6 & 9 & 8 & 15 & 21 & 37 & 20 \\
\hline $\begin{array}{l}\text { Médio } \\
\text { Incompleto }\end{array}$ & 222 & 8 & 2 & 8 & 11 & 8 & 13 & 19 & 9 & 34 & 76 & 34 \\
\hline $\begin{array}{l}\text { Médio } \\
\text { Completo }\end{array}$ & 333 & 6 & 6 & 7 & 8 & 12 & 16 & 25 & 19 & 56 & 124 & 54 \\
\hline $\begin{array}{l}\text { Superior } \\
\text { Incompleto }\end{array}$ & 22 & 1 & - & - & 1 & - & 1 & 1 & - & 4 & 7 & 7 \\
\hline $\begin{array}{l}\text { Superior } \\
\text { Completo }\end{array}$ & 10 & - & - & - & - & - & - & - & - & 1 & 6 & 3 \\
\hline Não se aplica & 0 & - & - & - & - & - & - & - & - & - & - & - \\
\hline Ignorado & 1.105 & 41 & 45 & 51 & 57 & 82 & 90 & 66 & 85 & 105 & 294 & 106 \\
\hline
\end{tabular}

Mulheres com o ensino médio completo foram apontadas em segundo lugar no grupo de maior taxa registrada, totalizando 333 casos registrados, sendo 124 (37,23\%) notificações em 2018 e 54 (16,21\%) em 2019. Foram 22 registros de mulheres diagnosticadas com SG tendo o ensino superior incompleto, sendo os maiores índices no ano de 2018 e 2019, ambos com os mesmos números, 7 (31\%).

É possível perceber que quanto maior o nível escolaridade, menor o número de mulheres gestantes diagnosticadas com sífilis. Obteve-se o total de 10 casos notificados entre os anos de 2009-2019 de mulheres com o ensino superior completo, sendo distribuídos entre 2017 (10\%), 2018, $6(60 \%)$, e 2019, 3 (30\%).

$\mathrm{O}$ acesso aos meios de prevenção e de tratamento está diretamente relacionado educacional institucional. Fica evidente, perante todos os estudos analisados, que fatores de risco individuais como gestantes adolescentes, múltiplos parceiros e baixa renda podem ter estreita relação com a disseminação da patologia nas classes com vulnerabilidade, contribuindo com a evasão escolar e a redução de oportunidades empregatícias. 
Tabela 5. Casos de gestantes com sífilis segundo classificação clínica por ano de diagnóstico. Recife, 2009_

\begin{tabular}{lcccccccccccc}
\hline $\begin{array}{l}\text { Classificação } \\
\text { Clínica }\end{array}$ & Total & $\mathbf{2 0 0 9}$ & $\mathbf{2 0 1 0}$ & $\mathbf{2 0 1 1}$ & $\mathbf{2 0 1 2}$ & $\mathbf{2 0 1 3}$ & $\mathbf{2 0 1 4}$ & $\mathbf{2 0 1 5}$ & $\mathbf{2 0 1 6}$ & $\mathbf{2 0 1 7}$ & $\mathbf{2 0 1 8}$ & $\mathbf{2 0 1 9}$ \\
$\begin{array}{l}\text { Sífilis } \\
\text { Primária }\end{array}$ & 639 & 42 & 32 & 18 & 25 & 42 & 53 & 60 & 45 & 82 & 194 & 46 \\
\hline $\begin{array}{l}\text { Sífilis } \\
\text { Secundária }\end{array}$ & 307 & 15 & 9 & 25 & 31 & 24 & 29 & 18 & 20 & 30 & 73 & 33 \\
\hline $\begin{array}{l}\text { Sífilis } \\
\text { Terciária }\end{array}$ & 221 & 4 & 5 & 7 & 14 & 19 & 18 & 22 & 28 & 29 & 50 & 25 \\
\hline $\begin{array}{l}\text { Sífilis } \\
\text { Latente }\end{array}$ & 323 & 8 & 7 & 7 & 8 & 3 & 12 & 9 & 20 & 49 & 148 & 52 \\
\hline Ignorado & 1.040 & 26 & 46 & 54 & 61 & 58 & 69 & 75 & 60 & 121 & 305 & 165 \\
\hline & Fonte: Sistema de Informação de Agravos de Notificação - SINAN & &
\end{tabular}

A sífilis primária e secundária possuem uma maior possibilidade de transmissão, devendo ser a patologia identificada preferencialmente no início da aquisição da doença. Um total de 639 casos de sífilis em estágio primário foram registrados em mulheres gestantes em Recife entre os anos de 2009-2019, sendo 2018 o ano de maior notificação, registrando 194 $(30,35 \%)$ novos casos e apresentando uma queda em 2019 de 148 casos, ano em que a taxa foi de $46(7,19 \%)$ gestantes infectadas.

No caso da sífilis secundária, foi registrada a menor taxa no ano de 2009 de gestantes infectadas no Recife, apenas 9 (2,93\%) casos, diferentemente do ano de 2018 que chegou a apresentar $73(23,77 \%)$ casos e novamente caiu para $33(10,74 \%)$ em 2019.

Na sífilis terciária, normalmente o indivíduo apresenta lesões cutâneas, cardiovasculares e/ou neurológicas, podendo levar o paciente a óbito quando sem tratamento. Assim, um total de 221 casos foram registrados entre 2009-2019, sendo 2018 o ano de maior notificação, 50 $(22,62 \%)$ casos, e 2019 o ano de queda, 25 (11,31\%) novos casos.

A sífilis latente, fase assintomática da doença, pode ser classificada como latente recente (quando o paciente tem menos de 2 anos de infecção) ou tardia (quando a infecção tem mais de 2 anos), podendo seu tempo variar de acordo com os sinais e sintomas característicos da fase secundária ou terciária. De 2009-2019, foi informado um total de 323 mulheres com SG, uma vez, o ano de 2018 apresentou o maior número de casos, 148 (45,82\%), e 2019 uma baixa, $52(16,09 \%)$ casos.

\section{CONCLUSÕES}

O levantamento das informações realizado através do registro de casos de Sífilis 
Gestacional no SINAN, revelou um incremento nas taxas de detecção de sífilis. Essas taxas podem ter sido impulsionadas pelo aumento na notificação dos casos, demonstrando que o monitoramento constante dos casos de sífilis em gestantes por meio do sistema de vigilância é essencial para que o município do Recife-PE se encaminhe para o cumprimento dos objetivos de eliminação da sífilis estabelecidos

Foi possível observar uma significativa redução dos casos de sífilis gestacional no ano de 2019 quando comparado ao ano de 2018. A diferença pode ser explicada como resultado da detecção e tratamento precoce associados as ações de educação em saúde, por outro lado, a diminuição dos casos em 2019 também pode ter sido resultado da subnotificação, o que corrobora para a falta de dados concretos para o estudo.

A redução dos casos de sífilis em gestante e a consequente diminuição da transmissão só serão possíveis de serem mantidas quando forem implementadas medidas de prevenção e controle eficientes. Para tanto, é de extrema importância que os profissionais da saúde e os gestores estejam envolvidos diretamente no desenvolvimento de estratégias, visando a melhoria da qualidade do serviço prestado às gestantes.

Dessa forma, o estudo permitiu observar que os fatores associados a ocorrência da sífilis gestacional foram, dentre outros fatores, a faixa etária, a idade gestacional, o nível de escolaridade, a raça/cor e a classificação clínica da doença. O perfil epidemiológico das mulheres afetadas pela sífilis gestacional no município do Recife-Pe, pode ter decorrido do início tardio do pré-natal associado as condições socioeconômicas das gestantes.

Nota-se, portanto, a necessidade da captação precoce e da adesão da gestante ao prénatal, assim como uma assistência de qualidade, de modo que as mulheres recebam atendidas pelos profissionais de saúde de acordo com suas especificidades e vulnerabilidades para que ações de promoção e prevenção sejam mais eficazes.

\section{REFERÊNCIAS}

ACOSTA, L. M. W; GONÇALVES, T. R.; BARCELLOS, N. T. Coinfecção HIV/sífilis na gestação e transmissão vertical do HIV: um estudo a partir de dados da vigilância epidemiológica. Rev Panam Salud Publica, v. 40, n. 6, p. 435-42, 2016.

ARAÚJO, L. R. L.; SILVA, V. C. C.; FILHO, P.S. G.; \& SOUSA, M. N. A. Preyalência de sífilis gestacional e congênita no estado de Goiás, Brasil. C\&D-Revista Eletrônica da FAINOR, v. 9. n. 2. p. 49-58, 2016.

AVELLEIRA, J. C. R.; BOTTINO, G. Sífilis: diagnóstico, tratamento e controle. An Bras Dermatol, v. 81, n. 2, p. 111-26, 2006. 
BERNARDES, F. et al. Sífilis em apresentação com fases sobrepostas: como conduzir. DST j. bras. doenças sex. transm, v. 24, n. 2, 2012.

BRASIL. Ministério da Saúde. Secretaria de Atenção à Saúde. Departamento de Atenção Básica. Caderno de Atenção Básica: Saúde Sexual e Saúde reprodutiva. Brasília - DF: 2013.

BRASIL. Ministério da Saúde. Secretaria de Vigilância em Saúde. Departamento de DST, Aids e Hepatites Virais. Boletim Epidemiológico - Sífilis. Brasília - DF, 2019.

BRASIL. Ministério da Saúde. Secretaria de Vigilância em Saúde. Departamento de DST, Aids e Hepatites Virais. Protocolo Clínico e Diretrizes Terapêuticas para Atenção Integral às Pessoas com Infecções Sexualmente Transmissíveis. Brasília - DF: 2015.

CARDOSO, A. R. P.; ARAÚJO, M. A. L. et al. Análise dos casos de sífilis gestacional e congênita nos anos de 2008 a 2010 em Fortaleza, Ceará, Brasil. Ciência \& Saúde Coletiva, v. 23. n. 2. p. 563-574, 2018.

CONCEIÇÃO, H. N. et al. Análise epidemiológica e espacial dos casos de sífilis gestacional e congênita. Saúde Debate, v. 4. n. 123. p. 1145-1158, 2019.

FIGUEIREDO, D. C. M. M. et al. Relação entre oferta de diagnóstico e tratamento da sífilis na atenção básica sobre a incidência de sífilis gestacional e congênita. Cad. Saúde Pública, v. 36. 3. p. 1-12, 2020.

FURTADO, M. F. S. et al. Fatores epidemiológicos da sífilis em gestantes no Município de São Luís-MA. Revista Uningá, v. 52 n. 1. p. 51-55, 2017.

GUANABARA, M. A. O. et al. Acolhimento e aconselhamento como tecnologias leves em saúde na prevenção da sífilis congênita em Fortaleza-Ceará. $1^{\circ}$ Congresso Internacional da Rede Unida, 2014.

LAZARINI, F. M.; BARBOZA, D. A. Intervenção educacional na Atenção Básica para prevenção da sífilis congênita. Rev. Latino-Am. Enfermagem, v. 25. e2845, 2017.

MACÊDO, V. C.; LIRA, P. I. C; FRIAS, P. G.; ROMAGUERA, L. M. D.; CAIRES, S. F. F.; XIMENES, R. A. A. Fatores de risco para sífilis em mulheres: estudo de caso-controle. Revista de Saúde Pública, v. 51, 2017.

OLIVEIRA, E. H. et al. Analysis of notificated cases of syphilis in pregnancy in the state of Paraiba, Brazil. Research, Society and Development, v. 9. n. 1. e179911900, 2020.

OLIVEIRA, R. B.; B.; PEIXOTO, A. M. C. L.; CARDOSO, M. D. Sífilis em gestantes adolescentes de Pernambuco. Adolesc. Saúde, v. 16, n. 2, p. 47-55. Rio de Janeiro, 201

RAMOS, M. G.; BONI, S. M. Prevalência da sífilis gestacional e congênita na população do município de Maringá - PR. Saúde e Pesquisa, v. 11. n. 3, 2018.

RAMPAZIO, I. T.; SOUZA, I. E. B.; CARVALHO, A. C. G. A atuação da enfermagem na prevenção e no tratamento da sífilis congênita. Revista Interdisciplinar do Pensamento Científico, v. 5. n. 3, 2019. 